\title{
Null geodesics in five-dimensional Reissner-Nordström anti-de Sitter black holes
}

\author{
P. A. González ${ }^{1, a}$, Marco Olivares ${ }^{1, b}$, Yerko Vásquez ${ }^{2, c}$, J. R. Villanueva ${ }^{3, d}$ \\ ${ }^{1}$ Facultad de Ingeniería y Ciencias, Universidad Diego Portales, Avenida Ejército Libertador 441, Santiago, Chile \\ ${ }^{2}$ Departamento de Física, Facultad de Ciencias, Universidad de La Serena, Avenida Cisternas 1200, La Serena, Chile \\ ${ }^{3}$ Instituto de Física y Astronomía, Universidad de Valparaíso, Avenida Gran Bretaña 1111, Valparaíso, Chile
}

Received: 8 October 2020 / Accepted: 5 March 2021 / Published online: 17 March 2021

(C) The Author(s) 2021

\begin{abstract}
The study of the motion of photons around massive bodies is one of the most useful tools to find the geodesic structure associated with said gravitational source. In the present work, different possible paths projected in an invariant hyperplane are investigated, considering a fivedimensional Reissner-Nordström anti-de Sitter black hole. Also, we study some observational tests, such as the bending of light and the Shapiro time delay effect. Mainly, we found that the motion of photons follows the hippopede of a Proclus geodesic, which is a new type of trajectory of the second kind, the Limaçon of Pascal being their analog geodesic in four-dimensional Reissner-Nordström anti-de Sitter black hole.
\end{abstract}

\section{Contents}

1 Introduction . . . . . . . . . . . . . . 1

2 Five-dimensional Reissner-Nordström anti-de Sitter black holes . . . . . . . . . . . . . . . 2

3 The null structure . . . . . . . . . . . . . . 3

3.1 Radial motion . . . . . . . . . . . . . . . . 4

3.2 Angular motion . . . . . . . . . . . . . 5

3.3 Bending of light . . . . . . . . . . . . 6

3.3.1 The deflection angle . . . . . . . . . 7

3.4 Second kind trajectories and hippopede geodesic 8

3.5 Critical trajectories and capture zone . . . . . . 8

3.6 Shapiro time delay . . . . . . . . . . . . . 9

4 Final remarks . . . . . . . . . . . . . . . . 9

References . . . . . . . . . . . . . . . . . 10

\footnotetext{
a e-mail: pablo.gonzalez@udp.cl

be-mail: marco.olivaresr@mail.udp.cl

c e-mail: yvasquez@userena.cl (corresponding author)

de-mail: jose.villanueva@uv.cl
}

\section{Introduction}

Extra-dimensional gravity theories have a long history, that begins with an original idea propounded by Kaluza \& Klein $[1,2]$ as a way to unify the electromagnetic and gravitational fields, and nowadays finds a new realization within modern string theory $[3,4]$. In spacetime dimensions $D \geq 4$, the spherically symmetric and static black hole solutions of general relativity in vacuum are known as SchwarzschildTangherlini black holes [5]. Additionally, the most natural extension of general relativity to higher dimensions that generates field equations of the second order is Lovelock gravity. Remarkably, the action contains terms that appear as corrections to the Einstein-Hilbert action in the context of string theory. In five spacetime dimensions the Lovelock lagrangian is given by the Einstein-Hilbert term and the Gauss-Bonnet term, which is quadratic in the curvature and it is a topological invariant in four dimensions. An exact black hole solution to the field equations of Einstein-Gauss-Bonnet theory was found in [6]. The geodesics of massive test particles in higher dimensional black hole spacetimes have been studied in Refs. [7-10], and it was shown that a particular feature of Reissner-Nordstrom spacetimes is that bound and escape orbits traverse through different universes, and the study of the motion of particles in five-dimensional spacetimes has been performed in Refs. [11-19].

The spacetime that we consider in this study is a generalization of the Reissner-Nordström anti-de Sitter (RNAdS) black hole to five dimensions, which is interesting in the context of the AdS/CFT correspondence [20-23]. A global five-dimensional Schwarzschild AdS solution was considered to describe a thermal plasma of finite extent expanding in a slightly anisotropic fashion [24]. Also, it was shown that four- and five-dimensional charged black holes in AdS spacetime could be obtained by compactifications of the type IIB supergravity in 11 dimensions. The properties of a Reissner- 
Nordstrom black hole in $d$-dimensional anti-de Sitter spacetime have been studied in Refs. [25,26], and the null geodesic structure of four-dimensional RNAdS black holes was analytically investigated in Ref. [27], where, concerning the radial motion, it was shown that the photons arrive at the event horizon in a finite proper time, and infinite coordinate time, similar to the Schwarzschild case. Also, concerning the angular motion of photons it was shown that there are five different kinds of motion for trapped photons, depending on the impact parameter of the orbits that corresponds to orbits where the photon arrives from infinity and falls into the event horizon, photons moving along the critical orbits that represent trajectories that come from infinity and fall asymptotically into a circle, photons falling from infinity arriving at some minimal distance and then going back to the infinity again, photon orbits described by Pascal Limaçon, which is an exclusive solution of a black hole with the cosmological constant but it does not depend on the value of the cosmological constant, and finally confined orbits for the photons.

The aim of this work is to study the null geodesics in a five-dimensional charged black hole, and to see if it is possible to find orbits for the motion of photons different from the previously mentioned ones for a RNAdS spacetime. Here, we will find the null structure geodesic analytically, and interestingly enough we find a new kind of orbit called "hippopede geodesics", which to the best of our knowledge is the first time that has been reported in the literature.

The five-dimensional spacetime considered allows us to study the role of extra dimensions, for instance, a fivedimensional Myers-Perry black hole spacetime was studied in Ref. [19], where the metric describes a spacetime with two spin parameters, and it was found that circular orbit geodesics are allowed, and the deflection angle and the strong deflection limit coefficients differ from four-dimensional Kerr black hole spacetime due to the presence of two spin parameters in the higher dimension. Another spacetime studied corresponds to a geometry described by a spherically symmetric four-dimensional solution embedded in a five-dimensional space, known as a brane-based spherically symmetric solution, analyzed in Ref. [16], where the authors found that the extra dimension contributes to the existence of bounded orbits for the photons, such as planetary and circular stable orbits. The spacetime considered in this work could be compared with four-dimensional RNAdS black holes, for the five-dimensional spacetime there is no additional parameter apart from the dimension added, but the event horizon is not the same due to the change in the lapse function, which could explain the differences between four- and five-dimensional spacetimes. However, as we will see, the effect of additional dimensions could be the existence of the hippopede of the Proclus geodesic found here, versus its analog geodesic in four-dimensional RNAdS black hole, i.e., the Limaçon of Pascal [27], both trajectories of the second kind.
It is worth mentioning that the same spacetime was considered in Ref. [11], where the null geodesics were studied from the point of view of the effective potential formalism and the dynamical systems approach. The radial and circular trajectories were investigated, and it was found that photons will trace out circular trajectories for only two distinct values of the specific radius of the orbits. The dynamical systems analysis was applied to determine the nature of trajectories and the fixed points, and it was shown that the null geodesics have a unique fixed point and these orbits are terminating orbits. Also, the thermodynamics and the stability of the spacetime under consideration were studied from a thermodynamic point of view, and there were found special conditions on the black hole mass and the black hole charge where the black hole is in stable phase [28].

The paper is organized as follows. In Sect. 2 we give a brief review of the spacetime considered. Then, in Sect. 3, we establish the null structure and we perform some test as the bending of light and the Shapiro time delay effect. Finally, we conclude in Sect. 4.

\section{Five-dimensional Reissner-Nordström anti-de Sitter black holes}

Schwarzschild and Reissner-Nordström black hole solutions in $d$ spacetime dimensions were presented by Tangherlini [5]. The five-dimensional RNAdS black holes are solutions of the equations of motion that arise from the action [26]

$S=-\frac{1}{16 \pi G_{5}} \int d^{5} x \sqrt{-g}\left(R-2 \Lambda-F^{2}\right)$,

where $G_{5}$ is the Newton gravitational constant in fivedimensional spacetime, $R$ is the Ricci scalar, $F^{2}$ represents the electromagnetic Lagrangian, and $\Lambda=-6 / \ell^{2}$ is the cosmological constant where $\ell$ is the radius of $\mathrm{AdS}_{5}$ space. The static and spherically symmetric metric that solves the field equation derived from the above action is given by

$\mathrm{d} s^{2}=-f(r) \mathrm{d} t^{2}+\frac{1}{f(r)} \mathrm{d} r^{2}+r^{2} \mathrm{~d} \Omega_{3}^{2}$,

where $f(r)$ for a $(n+1)$-dimensional RNAdS spacetime is the lapse function given by

$f(r)=1-\frac{m}{r^{n-2}}+\frac{q^{2}}{r^{2 n-4}}+\frac{r^{2}}{\ell^{2}}$,

where $m$ and $q$ are arbitrary constants, and $\mathrm{d} \Omega_{3}^{2}=\mathrm{d} \theta^{2}+$ $\sin ^{2} \theta \mathrm{d} \phi^{2}+\sin ^{2} \theta \sin ^{2} \phi \mathrm{d} \psi^{2}$ is the metric of the unit 3sphere. Also, $m$ is related to the ADM mass $\mathcal{M}$ of the spacetime through

$\mathcal{M}=\frac{(n-1) \omega_{n-1}}{16 \pi G} m$, 
where $\omega_{n-1}$ is the volume of the unit $(n-1)$-sphere. The parameter $q$ yields the charge

$\mathcal{Q}=\sqrt{2(n-1)(n-2)}\left(\frac{\omega_{n-1}}{8 \pi G}\right) q$.

In this work, we consider $n=4, m \rightarrow(2 M)^{2}$, and $q^{2} \rightarrow Q^{4}$, so the metric is

$f(r)=1-\left(\frac{2 M}{r}\right)^{2}+\left(\frac{Q^{2}}{r^{2}}\right)^{2}+\left(\frac{r}{\ell}\right)^{2} ;$

thereby, $M$ and $Q$ are related to the total mass $\mathcal{M}$ and the charge $\mathcal{Q}$ of the spacetime via the relations

$(2 M)^{2}=\frac{16 \pi G \mathcal{M}}{(n-1) \omega_{n-1}}, Q^{2}=\frac{8 \pi G \mathcal{Q}}{\sqrt{2(n-1)(n-2)} \omega_{n-1}}$.

This spacetime allows two horizons to occur (the event horizon $r_{+}$and the Cauchy horizon $r_{-}$), which are obtained from the equation $f(r)=0$, or

$P(r) \equiv r^{6}+\ell^{2} r^{4}-4 M^{2} \ell^{2} r^{2}+\ell^{2} Q^{4}=0$.

Now, with the change of variable $x=r^{2}-\ell^{2} / 3$, we obtain $P(x)=x^{3}-\alpha x+\beta$, where

$\alpha=\ell^{2}\left(4 M^{2}+\frac{\ell^{2}}{3}\right), \beta=\ell^{2}\left(Q^{4}+\frac{4 M^{2} \ell^{2}}{3}+\frac{2 \ell^{4}}{27}\right)$,

and the event and Cauchy horizons are given, respectively, by

$r_{+}=\sqrt{\xi_{0} \cos \xi_{1}-\frac{\ell^{2}}{3}}$,

$r_{-}=\sqrt{\frac{\xi_{0}}{2}\left(\sqrt{3} \sin \xi_{1}-\cos \xi_{1}\right)-\frac{\ell^{2}}{3}}$,

where $\xi_{0}=2 \sqrt{\alpha / 3}$ and $\xi_{1}=\frac{1}{3} \arccos \left(-\frac{3 \beta}{2} \sqrt{\frac{3}{\alpha^{3}}}\right)$. Also, the extremal black hole is characterized by the degenerate horizon $r_{e x t}=r_{+}=r_{-}$, which is obtained when

$4 \ell^{4}\left(4 M^{2}-Q^{2}\right)+8 \ell^{2} M^{2}\left(32 M^{4}-9 Q^{4}\right)-27 Q^{8}=0$.

In Fig. 1, we plot curves for different values of $Q$ that show the behavior of the lapse function against $r$, and we observe that when the charge of the black hole $Q$ increases we have a transition from a black hole to a naked singularity, passing by the extremal case.

Note that when $Q=0$ the lapse function reduces to the five-dimensional Schwarzschild anti-de Sitter black hole, and the spacetime allows one horizon to occur (the event horizon $r_{+}$) given by

$r_{+}=\ell \sinh \left[\frac{1}{2} \sinh ^{-1}\left(\frac{4 M}{\ell}\right)\right]$.

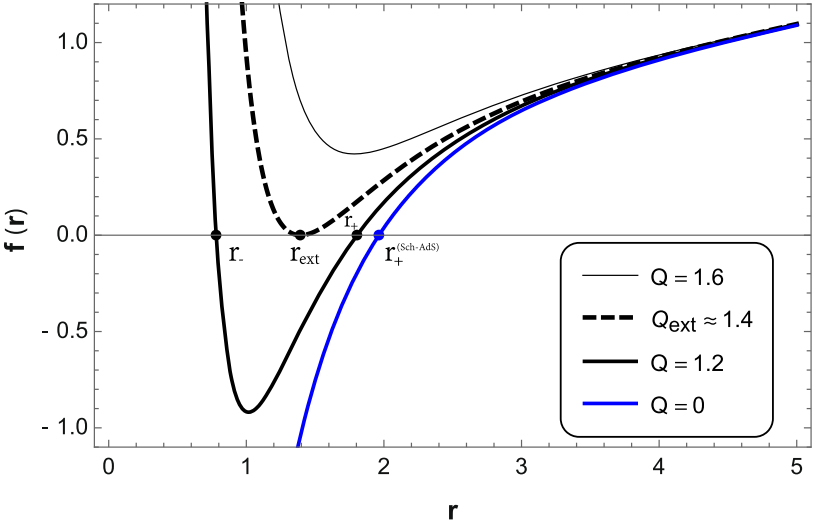

Fig. 1 The behavior of the metric function $f(r)$, with $M=1, \ell=10$, for different values of $Q$

In Fig. 1, the blue line corresponds to the case $Q=0$, and we observe that, for the same values of $\Lambda$ and $M$, the event horizon is greater for an uncharged than for a charged black hole.

\section{The null structure}

In order to obtain a description of the allowed motion in the exterior spacetime of the black hole, we use the standard Lagrangian formalism [29-31], so that the corresponding Lagrangian associated with the line element (2) reads

$\mathcal{L}=-\frac{f(r) \dot{t}^{2}}{2}+\frac{\dot{r}^{2}}{2 f(r)}+\frac{r^{2}}{2} \mathcal{L}_{\Omega}$,

where $\mathcal{L}_{\Omega}$ is the angular Lagrangian:

$\mathcal{L}_{\Omega}=\dot{\theta}^{2}+\sin ^{2} \theta \dot{\phi}+\sin ^{2} \theta \sin ^{2} \phi \dot{\psi}^{2}$,

and the dot indicates differentiation with respect to an affine parameter $\lambda$ along the geodesic. Since the Lagrangian (13) does not depend on the coordinates $(t, \psi)$, they are cyclic coordinates and, therefore, the corresponding conjugate momenta $\pi_{q}=\partial \mathcal{L} / \partial \dot{q}$ are conserved. Explicitly, we have

$$
\begin{aligned}
\pi_{t} & =-f(r) \dot{t} \equiv-E, \\
\pi_{\psi} & =r^{2} \sin ^{2} \theta \sin ^{2} \phi \dot{\psi}=L,
\end{aligned}
$$

where $E$ is a positive constant that describes the temporal invariance of the Lagrangian, which cannot be associated with energy because the spacetime defined by the line element (2) is not asymptotically flat, whereas the constant $L$ stands for the conservation of angular momentum, under which it is established that the motion is performed in an invariant hyperplane. Here, we claim to study the motion in the invariant hyperplane $\theta=\phi=\pi / 2$, so $\dot{\theta}=\dot{\phi}=0$ and, from Eq. (16), 
$\dot{\psi}=\frac{L}{r^{2}}$.

Therefore, using the fact that $\mathcal{L}=0$ for photons together with Eqs. (15) and (16), we obtain the following equations of motion:

$$
\begin{aligned}
& \left(\frac{\mathrm{d} r}{\mathrm{~d} \lambda}\right)^{2}=E^{2}-V^{2}(r), \\
& \left(\frac{\mathrm{d} r}{\mathrm{~d} t}\right)^{2}=\frac{f^{2}(r)}{E^{2}}\left[E^{2}-V^{2}(r)\right], \\
& \left(\frac{\mathrm{d} r}{\mathrm{~d} \psi}\right)^{2}=\frac{r^{4}}{L^{2}}\left[E^{2}-V^{2}(r)\right],
\end{aligned}
$$

where the effective potential $V^{2}(r)$ is defined by

$V^{2}(r) \equiv L^{2} \frac{f(r)}{r^{2}}=\frac{L^{2}}{\ell^{2}}+\frac{L^{2}}{r^{2}}-\frac{4 M^{2} L^{2}}{r^{4}}+\frac{Q^{4} L^{2}}{r^{6}}$.

The effective potential for the five-dimensional Schwarzschild anti-de Sitter black hole is obtained by setting $Q=0$ in the above equation.

\subsection{Radial motion}

For the radial motion the condition $L=0$ holds, which immediately yields a vanishing effective potential, $V^{2}=0$. Consequently, the equations governing this kind of motion are

$\frac{\mathrm{d} r}{\mathrm{~d} \lambda}= \pm E$

and

$\frac{\mathrm{d} r}{\mathrm{~d} t}= \pm f(r)$

where the sign $+(-)$ corresponds to massless particles moving toward spatial infinity (the event horizon). Assuming that photons are placed at $r=\bar{r}_{i}$ when $t=\lambda=0$, a straightforward integration of Eq. (22) yields

$\lambda(r)= \pm \frac{r-\bar{r}_{i}}{E}$

which is plotted in Fig. 2. We observed that with respect to the affine parameter the photons arrive at the horizon in a finite affine parameter, and when the photons move in the opposite direction, they require an infinite affine parameter to arrive at infinity, which does not depend on the charge of the black hole.

This behavior is essentially the same as that reported for the four-dimensional counterpart [27]. On the other hand, Eq. (23) can be rearranged and then integrated leading to the following expression:

$t(r)= \pm \ell^{2} \sum_{j=1}^{3} \delta_{j} t_{j}(r)$ where the functions $t_{j}(r)$ are given explicitly by

$t_{1}(r)=\ln \left|\frac{\bar{r}_{i}+r_{+}}{\bar{r}_{i}-r_{+}} \frac{r-r_{+}}{r+r_{+}}\right|$,

$t_{2}(r)=\ln \left|\frac{\bar{r}_{i}+r_{-}}{\bar{r}_{i}-r_{-}} \frac{r+r_{-}}{r+r_{-}}\right|$,

$t_{3}(r)=\tan ^{-1}(r / R)-\tan ^{-1}\left(\bar{r}_{i} / R\right)$,

with the corresponding constants,

$\delta_{1}=\frac{r_{+}^{3}}{2\left(r_{+}^{2}-r_{-}^{2}\right)\left(r_{+}^{2}+R^{2}\right)}$,

$\delta_{2}=\frac{-r_{-}^{3}}{2\left(r_{+}^{2}-r_{-}^{2}\right)\left(r_{-}^{2}+R^{2}\right)}$,

$\delta_{3}=\frac{R^{3}}{\left(r_{+}^{2}+R^{2}\right)\left(r_{-}^{2}+R^{2}\right)}$,

$R=\sqrt{\ell^{2}+r_{+}^{2}+r_{-}^{2}}$.

Thus, an observer located at $\bar{r}_{i}$ will measure an infinite time for the photon to reach the event horizon, which also occurs in $3+1$ dimensions. Nevertheless, when the test particles move in the opposite direction, they require a finite coordinate time to arrive at infinity, given by the relation

$t_{\infty}=\lim _{r \rightarrow \infty} t(r)$

or, explicitly (with $\tilde{t}_{\infty} \equiv t_{\infty} / \ell^{2}-\delta_{3} \pi / 2$ )

$\tilde{t}_{\infty}=\delta_{1} \ln \left|\frac{\bar{r}_{i}+r_{+}}{\bar{r}_{i}-r_{+}}\right|+\delta_{2} \ln \left|\frac{\bar{r}_{i}+r_{-}}{\bar{r}_{i}-r_{-}}\right|-\delta_{3} \arctan \left(\frac{\bar{r}_{i}}{R}\right)$.

All previously described by Eqs. (24) and (25) is shown in Fig. 2. It is interesting to note that the behavior given in (33) also appears in Lifshitz spacetimes [32,33], where it was argued that this corresponds to a general behavior of these manifolds [34], and also occurs in the three-dimensional rotating Hořava-AdS black hole [35].

On the other hand, for $Q=0 \mathrm{Eq}$. (24) is valid. However, the solution for the coordinate time is given by

$$
\begin{aligned}
t(r) & = \pm \ell^{2} \sum_{j=1}^{2} \zeta_{j} t_{j}(r) \\
t_{1}(r) & =\ln \left|\frac{\bar{r}_{i}+r_{+}}{\bar{r}_{i}-r_{+}} \frac{r-r_{+}}{r+r_{+}}\right|, \\
t_{2}(r) & =\tan ^{-1}\left[\frac{r}{\left(r_{+}^{2}+\ell^{2}\right)^{\frac{1}{2}}}\right]-\tan ^{-1}\left[\frac{\bar{r}_{i}}{\left(r_{+}^{2}+\ell^{2}\right)^{\frac{1}{2}}}\right],
\end{aligned}
$$




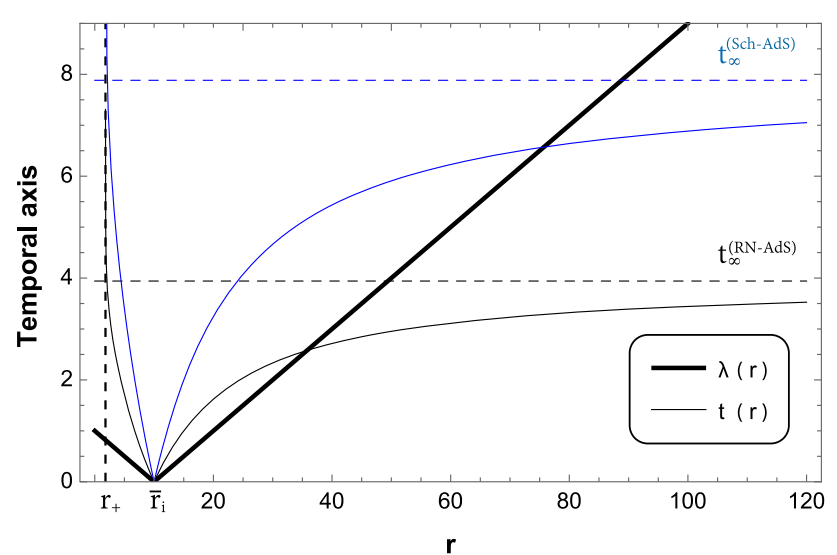

Fig. 2 Plot of the radial motion of massless particles. Particles moving to the event horizon, $r_{+}$, cross it with a finite affine parameter, but an external observer will see that photons take an infinite (coordinate) time to do it. Here we have used the values $E=100, \ell=10$, and $\bar{r}_{i}=10$. Black-thin line for $Q=1.2$, and $r_{+} \approx 1.80$. Blue line for $Q=0$, and $r_{+} \approx 1.96$

and

$\zeta_{1}=\frac{r_{+}}{2\left(2 r_{+}^{2}+\ell^{2}\right)}$,

$\zeta_{2}=\frac{\left(r_{+}^{2}+\ell^{2}\right)^{\frac{1}{2}}}{2 r_{+}^{2}+\ell^{2}}$.

Also, in the asymptotic region, $r \rightarrow \infty$, the time to arrive at infinity reduces to

$t_{\infty}=\ell^{2} \zeta_{1} \ln \left|\frac{\bar{r}_{i}+r_{+}}{\bar{r}_{i}-r_{+}}\right|+\ell^{2} \zeta_{2}\left[\frac{\pi}{2}-\tan ^{-1}\left(\frac{\bar{r}_{i}}{R}\right)\right]$.

It is possible to observe in Fig. 2 that an observer located at $\bar{r}_{i}$ will measure an infinite coordinate time for the photon to reach the event horizon, and it does not depend on the charge of the black hole. However, when the photons move in the opposite direction, they require a finite coordinate time to arrive at infinity, which decreases with the charge of the black hole.

\subsection{Angular motion}

Now we study the motion with $L \neq 0$, so we put our attention in Eq. (20), which, after using (21), is conveniently written as

$$
\begin{aligned}
\left(r \frac{\mathrm{d} r}{\mathrm{~d} \psi}\right)^{2} & =\left(\frac{1}{b^{2}}-\frac{1}{\ell^{2}}\right) r^{6}-r^{4}+4 M^{2} r^{2}-Q^{4} \\
& =\frac{r^{6}}{\mathcal{B}^{2}}-r^{4}+4 M^{2} r^{2}-Q^{4}
\end{aligned}
$$

where $b \equiv L / E$ is the impact parameter and $\mathcal{B}$ is the anomalous impact parameter, which is a typical quantity of the anti-de Sitter spacetimes [30].

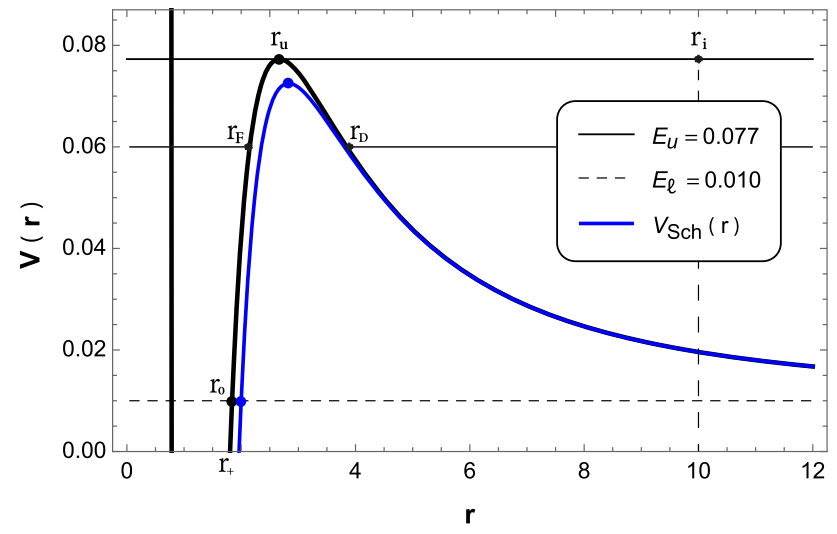

Fig. 3 Plot of the effective potential of photons. Here we have used the values $L=1, M=1, \ell=10, r_{i}=10, Q=1.2$ (black line), and $Q=0$ (blue line)

In a first approach, it is necessary to perform a qualitative analysis of the effective potential. So, we can observe in Fig. 3 the existence of a maximum potential located at

$r_{u}=2 \sqrt{2} M \cos \left[\frac{1}{2} \sin ^{-1}\left(\frac{\sqrt{3} Q^{2}}{4 M^{2}}\right)\right]$.

Therefore, $E_{u}$ is given by $V\left(r_{u}\right)$, and it corresponds to the energy of the photons for which the potential is maximum. Also, it is possible to define $E_{\ell}$ given by $V(r \rightarrow \infty)=$ $L / \ell$, and $b_{\ell}=L / E_{\ell}=\ell$ as its impact parameter. Thus, for orbits of the first kind, the parameter $b_{\ell}$ is not allowed for photons, and the deflection of the light is allowed for $E_{\ell}<E<E_{u}\left(b_{u}<b<\ell\right)$, see Fig. 3; thereby, the radius of $\mathrm{AdS}_{5}$ space $\ell$ physically corresponds to an impact parameter that the photons cannot reach. On the contrary, for orbits of the second kind, with $0<E<E_{u}\left(b_{u}<b<\infty\right)$, the photons can have an impact parameter $\ell$ by describing the hippopede geodesic, with a return point $r_{0}$, see Fig. 3 . Note that for $Q=0, r_{u}(Q=0)=2 \sqrt{2} M$, and it is greater than $r_{u}$ for RNAdS; however, the maximum value of the potential $E_{u}(Q=0)$ is smaller than $E_{u}$ for RNAdS. Also, the $r_{0}$ value is the same for the two spacetimes.

Next, based on the impact parameter values and Fig. 3, we present a brief qualitative description of the allowed angular motions for photons in RNAdS.

- Capture zone: If $0<b<b_{u}$, photons fall inexorably to the horizon $r_{+}$, or escape to infinity, depending on the initial conditions, and its cross section, $\sigma$, in this geometry is [36]

$$
\sigma=\pi b_{u}^{2}=\frac{\pi r_{u}^{2}}{f\left(r_{u}\right)}
$$


- Critical trajectories: If $b=b_{u}$, photons can stay in one of the unstable inner circular orbits of radius $r_{u}$. Therefore, the photons that arrive from the initial distance $r_{i}\left(r_{+}<\right.$ $r_{i}<r_{u}$, or $r_{u}<r_{i}<\infty$ ) can asymptotically fall to a circle of radius $r_{u}$. The affine period in such orbit is

$T_{\lambda}=\frac{2 \pi r_{u}^{2}}{L}$

and the coordinate period is

$$
T_{t}=2 \pi b_{u}=\frac{2 \pi r_{u}}{\sqrt{f\left(r_{u}\right)}} .
$$

- Deflection zone. If $b_{u}<b<b_{\ell}$, this zone presents orbits of the first and the second kind. The orbits of the first kind are allowed in the interval $r_{D} \leq r<\infty$, where the photons can come from a finite distance or from an infinity distance until they reach the distance $r=r_{D}$ (which is a solution of the equation $V\left(r_{D}\right)=E$ ), and then the photons are deflected. Note that photons with $b \geq b_{\ell}=\ell$ are not allowed in this zone. The orbits of the second kind are allowed in the interval $r_{+}<r \leq r_{F}$, where the photons come from a distance greater than the event horizon, then they reach the distance $r_{F}$ (which is a solution of the equation $V\left(r_{F}\right)=E$ ) and then they plunge into the horizon.

- Second kind and hippopede geodesic. If $b_{u}<b<\infty$, the return point is in the range $r_{+}<r<r_{u}$, and then the photons plunge into the horizon. However, when $b=b_{\ell}$ a special geodesic can be obtained, known as the hippopede of Proclus.

On the other hand, it was argued that an introduction of a negative tidal charge in four-dimensional ReissnerNordström black holes can describe black hole solutions in theories with extra dimensions in Ref. [19]. Also, by considering a naked singularity, i.e., $q=Q^{2} / M^{2}>1$ the existence was shown of a critical value of $q=q_{c}=9 / 8$ for a shadow existence; thereby, for $q \leq 9 / 8$ the ReissnerNordström spacetimes have shadows and the radius of the last unstable circular orbit is $r_{u}=3 M / 2$, while for $q>9 / 8$ the shadows do not exist. Interestingly, at the same critical value the quasinormal modes for the scattering exhibit a different behavior [37]. It is responsible for the existence of circular orbits of neutral test particles [38]. The critical charge $q_{c}$ arises from the last unstable circular orbits considering a naked singularity; thus, for five-dimensional RNAdS spacetimes, from Eq. (41), one can deduce the critical value of the charge where the last unstable circular orbit occurs, given by $Q_{c}^{2}=\frac{4 M^{2}}{\sqrt{3}}$, so $q_{c}=\frac{4}{\sqrt{3}}$, and the radius of the last unstable circular orbit is $r_{u}=2 M$. Also for $Q>Q_{\text {ext }}$, where

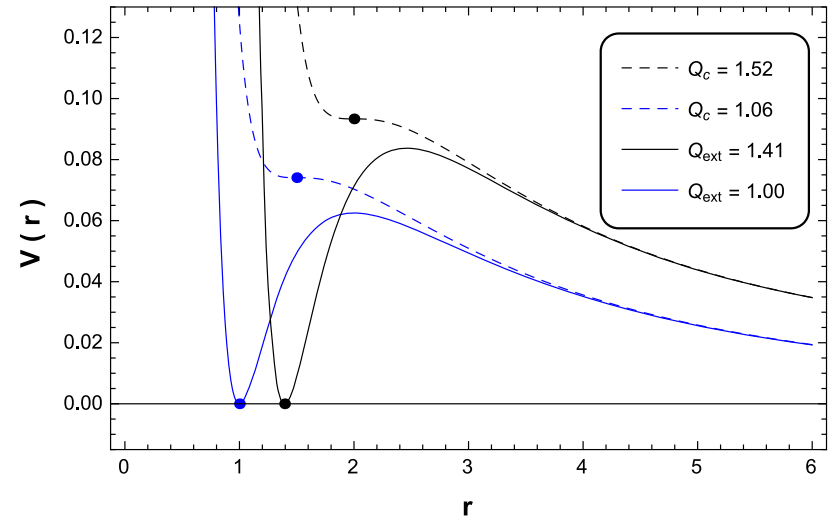

Fig. 4 Plot of the effective potential of photons as a function of $r$ for different values of the charge $Q$. Here we have used the values $L=1$, and $M=1$. Blue line corresponds to four-dimensional RN spacetime $Q_{\text {ext }}=1.0 r_{+}=1, Q_{c}=1.06$, and the radius of the last unstable circular orbit is $r_{u}=1.5$. Black line corresponds to five-dimensional RN AdS spacetime with $\ell=10, Q_{\text {ext }}=1.41, r_{+}=1.39, Q_{c}=1.52$, and the radius of the last unstable circular orbit is $r_{u}=2$

$$
Q_{\text {ext }}=\left[\frac{2 \ell}{27}\left[\left(\ell^{2}+12 M^{2}\right)^{3 / 2}-\ell\left(\ell^{2}+18 M^{2}\right)\right]\right]^{1 / 4},
$$

the spacetime describes a naked singularity, where $Q_{\text {ext }}$ was obtained using Eq. (12). It is worth noticing that $q_{c}$ does not depend on the cosmological constant. However, the value of the charge for which the spacetime describes a naked singularity depends on the value of the cosmological constant. In Fig. 4, we show the behavior of the effective potential as a function of $r$, where the points indicate the radius of the last unstable circular orbit for the critical charge $q_{c}$ and the event horizon for the extremal charge $Q_{\text {ext }}$. We observe that the critical charge $q_{c}, Q_{\text {ext }}$, and the radius of the last unstable circular orbit increase when the spacetime is the five-dimensional RNAdS instead of the four-dimensional Reissner-Nordström spacetime.

\subsection{Bending of light}

Now, in order to obtain the bending of light we consider Eq. (40), which can be written as

$$
\left(r \frac{\mathrm{d} r}{\mathrm{~d} \psi}\right)^{2}=\frac{r^{6}-\mathcal{B}^{2} r^{4}+4 M^{2} \mathcal{B}^{2} r^{2}-Q^{4} \mathcal{B}^{2}}{\mathcal{B}^{2}}=\frac{\mathcal{P}(r)}{\mathcal{B}^{2}} .
$$

Thus, in order to obtain the return points, we solve the equation $\mathcal{P}(r)=0$. Thus, we perform the change of variable $y=r^{2}+\mathcal{B}^{2} / 3, \mathcal{P}(y)=y^{3}-\tilde{\alpha} y-\tilde{\beta}$, where

$\tilde{\alpha}=\mathcal{B}^{2}\left(\frac{\mathcal{B}^{2}}{3}-4 M^{2}\right), \quad \tilde{\beta}=\mathcal{B}^{2}\left(Q^{4}-\frac{4 M^{2} \mathcal{B}^{2}}{3}+\frac{2 \mathcal{B}^{4}}{27}\right)$, 
and the deflection distance $r_{D}$ is given by

$r_{D}=\sqrt{\chi_{0} \cos \chi_{1}+\frac{\mathcal{B}^{2}}{3}}$,

and the return point $r_{F}$ is

$r_{F}=\sqrt{\frac{\chi_{0}}{2}\left(\sqrt{3} \sin \chi_{1}-\cos \chi_{1}\right)+\frac{\mathcal{B}^{2}}{3}}$,

where $\chi_{0}=2 \sqrt{\tilde{\alpha} / 3}$ and $\chi_{1}=\frac{1}{3} \arccos \left(\frac{3 \tilde{\beta}}{2} \sqrt{\frac{3}{\tilde{\alpha}^{3}}}\right)$.

Then, after a brief manipulation, and performing the change of variable $r=\mathcal{B} \sqrt{4 x+1 / 3}$ it is possible to integrate Eq. (46), given the following expression:

$\psi=\int_{x_{D}}^{x} \frac{\mathrm{d} x}{2 \sqrt{4 x^{3}-g_{2} x-g_{3}}}$,

where the invariants are given by

$g_{2}=\frac{1}{12}-\frac{M^{2}}{\mathcal{B}^{2}}$

$g_{3}=\frac{1}{16}\left(\frac{2}{27}-\frac{4 M^{2}}{3 \mathcal{B}^{2}}+\frac{Q^{4}}{\mathcal{B}^{4}}\right)$.

Therefore, by integrating Eq. (50) and then solving for $r$ leads to

$r(\psi)=\mathcal{B} \sqrt{4 \wp\left(2 \psi+\omega_{D}\right)+1 / 3}$,

where $\omega_{D}=\wp^{-1}\left(r_{D}^{2} / 4 \mathcal{B}^{2}-1 / 12\right)$. In Fig. 5 we show the behavior of the bending of light. We observe that the deflection angle is greater, when the black hole is uncharged. On the other hand, note that the above equations are straightforwardly obtained for five-dimensional Schwarzschild-anti-de Sitter spacetime.

\subsubsection{The deflection angle}

It is well known that photons can escape to infinity during a scattering process. So, by considering $\left.r(\psi)\right|_{\psi=0}=r_{D}$, the shortest distance to the black hole at which the deflection happens, and assuming that the incident photons are coming from infinity and escape to infinity, we have $\left.r(\psi)\right|_{\psi=\psi_{\infty}}=$ $\infty$. Now, by using Eq. (53) we obtain $2 \psi_{\infty}=-\omega_{D}$, and the deflection angle, $\hat{\alpha}=2 \psi_{\infty}-\pi$, is given by

$\hat{\alpha}=-\wp^{-1}\left(r_{D}^{2} / 4 \mathcal{B}^{2}-1 / 12\right)-\pi$.

The evolution of the deflection angle has been plotted in Fig. 6 which shows an asymptotic behavior as $E \rightarrow E_{u}$. We can observe that the deflection angle takes an infinite value when $E=E_{u}$, such that $E_{u}$ increases when the charge of the black hole increases.

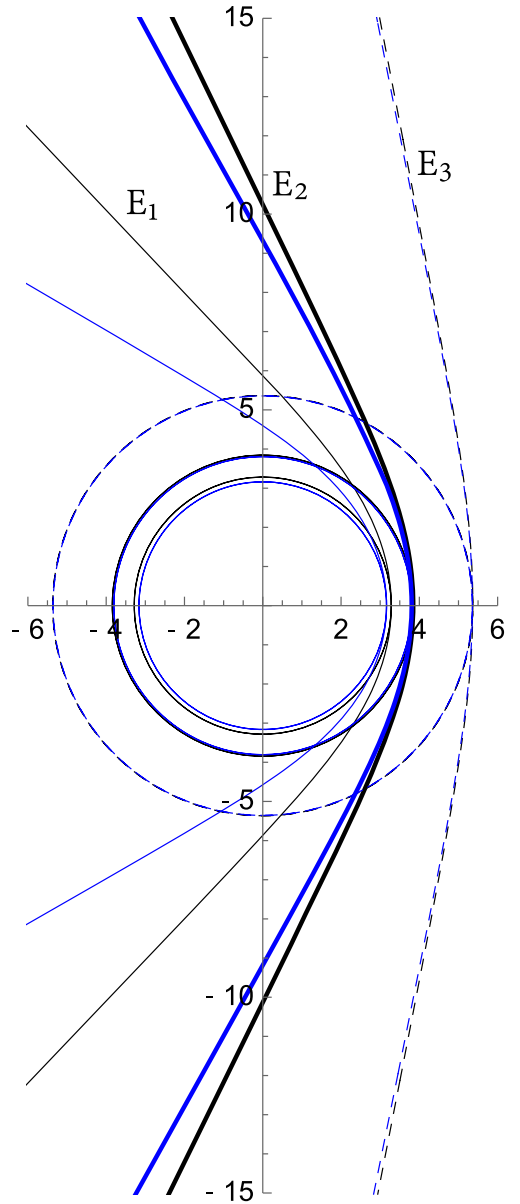

Fig. 5 Polar plot for deflection of light with $\ell=10$, and $L=1$. Thin line $E_{1}=0.07$, thick line $E_{2}=0.06$, and dashed line $E_{3}=0.04$. Black lines for $Q=1.20$ and blue lines for $Q=0$

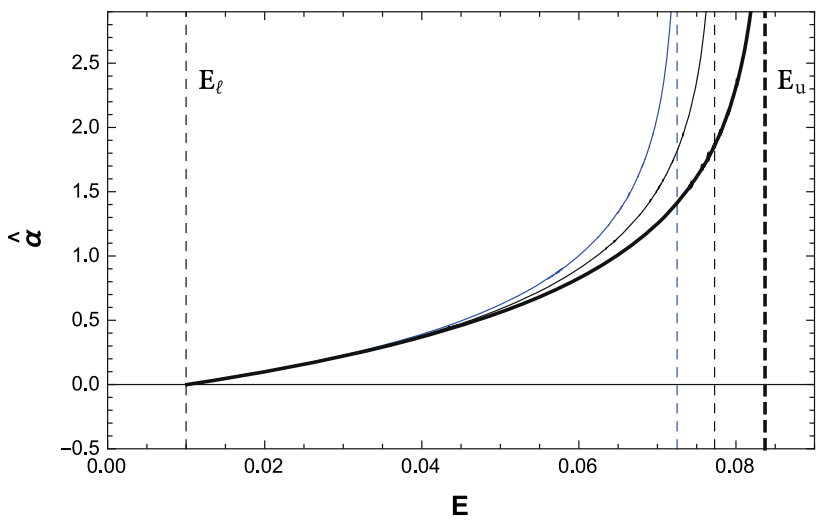

Fig. 6 The behavior of the deflection angle $\hat{\alpha}$ in terms of $E$, demonstrated for $L=1, M=1$ and $\ell=10$. Black-thin line for $Q=1.2$, black-thick line $Q=Q_{\text {ext }}=1.40$, and blue line for $Q=0$. As expected, the deflection angle reaches its limit as $E$ tends to $E_{u}$ which for $Q=1.20$ is around 0.077 , for $Q=Q_{\text {ext }}$ is 0.084 and it is 0.073 for $Q=0$ 


\subsection{Second kind trajectories and hippopede geodesic}

The spacetime allows for second kind trajectories, when $b_{u}<$ $b<\infty$, where the return point is in the range $r_{+}<r<r_{u}$, and then the photons plunge into the horizon. However, a special geodesic can be obtained when the anomalous impact parameter $\mathcal{B} \rightarrow \infty(b=\ell)$. In this case, the radial coordinate is restricted to $r_{+}<r<r_{0}$, and the equation of motion (40) can be written as

$\psi=-\int_{r_{0}}^{r} \frac{r \mathrm{~d} r}{\sqrt{-r^{4}+4 M^{2} r^{2}-Q^{4}}}$,

and the return points are

$$
\begin{aligned}
& r_{0}=2 M \cos \left[\frac{1}{2} \sin ^{-1}\left(\frac{Q^{2}}{2 M^{2}}\right)\right], \\
& \rho_{0}=2 M \sin \left[\frac{1}{2} \sin ^{-1}\left(\frac{Q^{2}}{2 M^{2}}\right)\right] .
\end{aligned}
$$

Thus, it is straightforward to find the solution of Eq. (55), which is given by

$r(\psi)=\sqrt{2 M^{2}+\sqrt{4 M^{4}-Q^{4}} \cos [2 \psi]}$,

which represents the hippopede of the Proclus geodesic (see Fig. 7) [39]. This trajectory is a new type of orbit in fivedimensional RNAdS, and it does not depend on the value of the cosmological constant. It is worth mentioning that the analog geodesic in four-dimensional RNAdS corresponds to the Limaçon of Pascal [27]. Also, when the spacetime is the five-dimensional Schwarzschild-anti-de Sitter spacetime this geodesic is given by $r=2 M \cos [\psi]$, which describes a circumference with radius $M$ that is analog to the cardioid geodesics found in four-dimensional Schwarzschild-anti-de Sitter spacetime [30].

\subsection{Critical trajectories and capture zone}

In the case of $b=b_{u}$, the particles can be confined on unstable circular orbits of the radius $r_{u}$. This kind of motion is indeed ramified into two cases; critical trajectories of the first kind (CFK) in which the particles come from a distant position $r_{i}$ to $r_{u}$ and those of the second kind (CSK) where the particles start from an initial point $d_{i}$ at the vicinity of $r_{i}$ and then tend to this radius by spiraling. We obtain the following equations of motion for the aforementioned trajectories:

$r(\psi)=\left[\rho_{u}^{2}+\left(r_{u}^{2}-\rho_{u}^{2}\right)\left(\frac{1+C e^{\kappa \psi}}{1-C e^{\kappa \psi}}\right)^{2}\right]^{1 / 2}$,

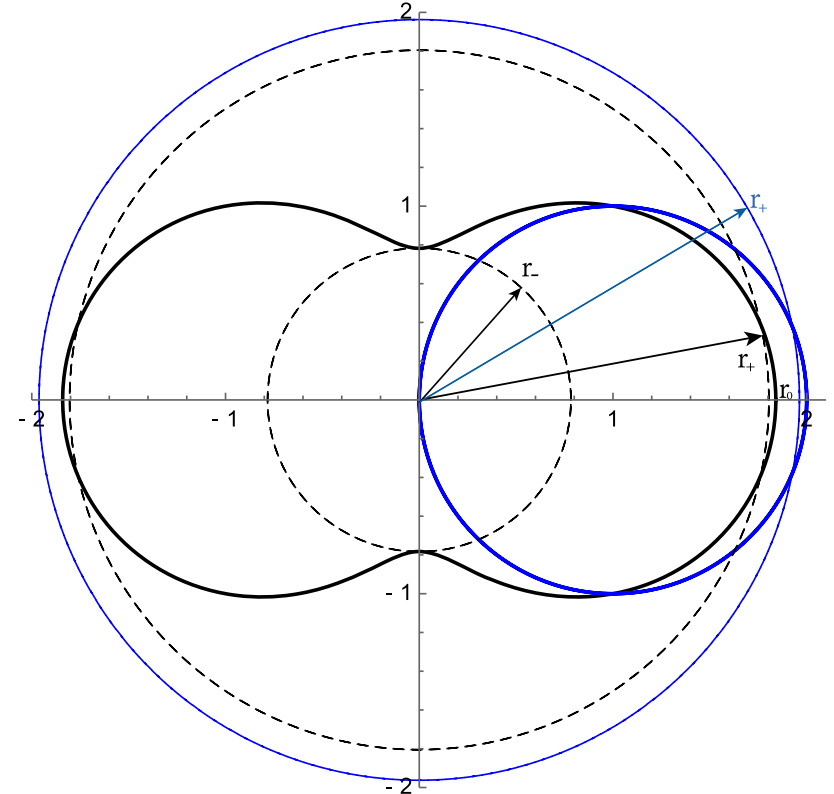

Fig. 7 The hippopede geodesic (black line), with $E_{\ell}=0.01, Q=$ 1.20 , and $\ell=10$, dashed black lines correspond to the horizons. The circumference geodesic (blue lines), with $E_{\ell}=0.01, Q=0$, and $\ell=10$

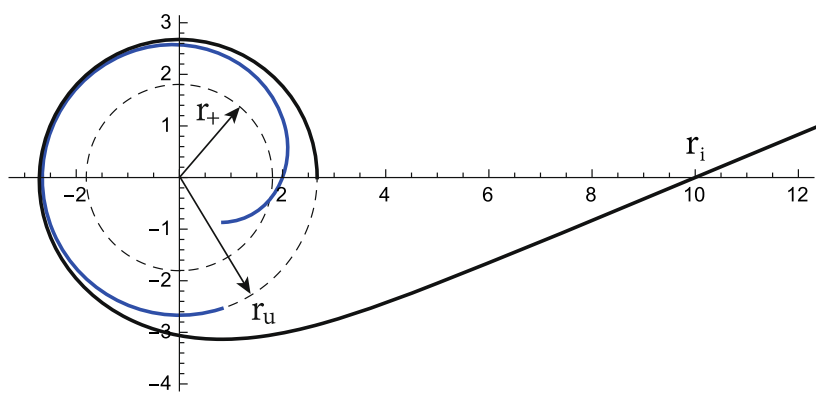

Fig. 8 The critical trajectories $r(\psi)$ plotted for $Q=1.2, L=1.0$ $\ell=10$, with $E_{u} \approx 0.077, r_{u} \approx 2.67$, and $r_{i}=10$. Black line for CFK and blue line for CSK trajectories

where

$\rho_{u}=\sqrt{\mathcal{B}_{u}^{2}-2 r_{u}^{2}}$,

$C=\left|\frac{\sqrt{r_{i}^{2}-\rho_{u}^{2}}-\sqrt{r_{u}^{2}-\rho_{u}^{2}}}{\sqrt{r_{i}^{2}-\rho_{u}^{2}}+\sqrt{r_{u}^{2}-\rho_{u}^{2}}}\right|$,

$\kappa=\frac{2 \sqrt{r_{u}^{2}-\rho_{u}^{2}}}{\mathcal{B}_{u}}$

In Fig. 8, we show the behavior of the CFK and CSK trajectories, given by Eq. (59). Note that, for trajectories of the second kind, $r_{i}$ must be replaced by $d_{i}$ in the constant $C$ (61). On the other hand, for photons with an impact parameter smaller than the critical one $\left(b_{u}\right)$, which are in the capture zone, they can plunge into the horizon or escape to infinity, with a cross section given by Eq. (42). 


\subsection{Shapiro time delay}

An interesting relativistic effect in the propagation of light rays is the apparent delay in the time of propagation for a light signal passing near the Sun, which is a relevant correction for astronomic observations, and is called the Shapiro time delay. The time delay of radar echoes corresponds to the determination of the time delay of radar signals which are transmitted from the Earth through a region near the Sun to another planet or spacecraft and then reflected back to the Earth. The time interval between emission and return of a pulse as measured by a clock on the Earth is

$t_{12}=2 t\left(r_{1}, r_{D}\right)+2 t\left(r_{2}, r_{D}\right)$,

where $r_{D}$ is the closest approach to the Sun. Now, in order to calculate the time delay we use Eq. (18), and by considering that $\mathrm{d} r / \mathrm{d} t$ vanishes, we have $\frac{E^{2}}{L^{2}}=\frac{f\left(r_{D}\right)}{r_{D}^{2}}$. Thus, the coordinate time that the light requires to go from $r_{D}$ to $r$ is given by

$t\left(r, r_{D}\right)=\int_{r_{D}}^{r} \frac{\mathrm{d} r}{f(r) \sqrt{1-\frac{r_{D}^{2}}{f\left(r_{D}\right)} \frac{f(r)}{r^{2}}}}$.

So, at first order correction we obtain

$t\left(r, r_{D}\right)=\sqrt{r^{2}-r_{D}^{2}}+t_{M}(r)+t_{Q}(r)+t_{\ell}(r)$,

where

$$
\begin{aligned}
t_{M}(r) & =\frac{6 M^{2}}{r_{D}} \sec ^{-1}\left(\frac{r}{r_{D}}\right), \\
t_{Q}(r) & =-\frac{Q^{4}}{4 r_{D}^{3}}\left[5 \sec ^{-1}\left(\frac{r}{r_{D}}\right)+\frac{3 r_{D} \sqrt{r^{2}-r_{D}^{2}}}{r^{2}}\right], \\
t_{\ell}(r) & =-\frac{\sqrt{r^{2}-r_{D}^{2}}}{3 \ell^{2}}\left(r^{2}+\frac{r_{D}^{2}}{2}\right) .
\end{aligned}
$$

Therefore, for the circuit from point 1 to point 2 and back the delay in the coordinate time is

$$
\Delta t:=2\left[t\left(r_{1}, r_{D}\right)+t\left(r_{2}, r_{D}\right)-\sqrt{r_{1}^{2}-r_{D}^{2}}-\sqrt{r_{2}^{2}-r_{D}^{2}}\right],
$$

where

$$
\begin{aligned}
\Delta t= & 2\left[t_{M}\left(r_{1}\right)+t_{M}\left(r_{2}\right)+t_{Q}\left(r_{1}\right)+t_{Q}\left(r_{2}\right)\right] \\
& +2\left[t_{\ell}\left(r_{1}\right)+t_{\ell}\left(r_{2}\right)\right] .
\end{aligned}
$$

Now, for a round trip in the solar system, we have $\left(r_{D}<<\right.$ $\left.r_{1}, r_{2}\right)$

$$
\begin{aligned}
\Delta t \approx & {\left[\frac{12 M^{2}}{r_{D}}-\frac{5 Q^{4}}{2 r_{D}^{3}}\right]\left[\sec ^{-1}\left(\frac{r_{1}}{r_{D}}\right)+\sec ^{-1}\left(\frac{r_{2}}{r_{D}}\right)\right] } \\
& -\frac{2}{3 \ell^{2}}\left(r_{1}^{3}+r_{2}^{3}\right) .
\end{aligned}
$$

Note that the classical result of GR is $\Delta t_{G R}=4 M_{\odot}[1+\ln$ $\left.\left(\frac{4 r_{1} r_{2}}{r_{D}^{2}}\right)\right]$. For a round trip from the Earth to Mars and back, we find (for $\left.r_{D} \ll r_{1}, r_{2}\right) r_{1} \approx r_{2}=2.25 \times 10^{8} \mathrm{~km}$ to be the average Earth-Mars distance. Considering $r_{D}$ as the closest approach to the Sun, like the radius of the $\operatorname{Sun}\left(R_{\odot} \approx\right.$ $696,000 \mathrm{~km})$ plus the solar corona $\left(\sim 10^{6} \mathrm{~km}\right), r_{D} \approx 1.696 \times$ $10^{6} \mathrm{~km}$, then the time delay is $\Delta t_{G R} \approx 240 \mu \mathrm{s}$. On the other hand, if we consider the limit $M \rightarrow M_{\odot}, Q=0$, and $\Lambda=0$, in Eq. (71), we obtain $\Delta t \approx 161 \mathrm{~ns}$. It is worth to mention that this value is closer to the value measured in the Viking mission, where the error in the time measurement of a circuit was only about $10 \mathrm{~ns}$ [40].

\section{Final remarks}

We considered the motion of photons in the background of five-dimensional RNAdS black holes, and we established the null structure geodesic. This spacetime is described by one Cauchy horizon and an event horizon. Concerning the radial motion, we showed that, as seen by a system external to the photons, they will fall asymptotically to the event horizon. On the other hand, this external observer will see that photons arrive in a finite coordinate time to spatial infinity. Concerning the angular motion, we found analytically the orbit of the first and the second kind; and also the critical orbit. Interestingly, for the trajectory of the second kind, we found that the motion of photons follows the hippopede of the Proclus geodesic when the parameter of impact $b$ takes the value $b=\ell$, and it does not depend on the value of the cosmological constant, the Limaçon of Pascal being their analog geodesic in four-dimensional RNAdS. On the other hand, we studied some observational test such as the bending of light, which show a similar behavior to the four-dimensional RNAdS, and the Shapiro time delay effect, where our results show that $\Delta t \approx 161 \mathrm{~ns}$ while for GR $\Delta t_{G R} \approx 240 \mu \mathrm{s}$.

Also, by comparing five-dimensional RNAdS black holes with four-dimensional RNAdS black holes, for the fivedimensional spacetime there is not an additional parameter apart from the dimension added, contrary to a fivedimensional Myers-Perry black hole spacetime, where the metric describes a spacetime with two spin parameters which could explain the differences with respect to the fourdimensional Kerr black hole spacetime due to the pres- 
ence of two spin parameters in higher dimension. For fivedimensional RNAdS black holes the event horizon is not the same due to the change in the lapse function, which could explain the differences between four- and five-dimensional spacetimes. However, the effect of an additional dimension could be the existence of the hippopede of the Proclus geodesic found here, versus its analog geodesic in fourdimensional RNAdS black hole, i.e., the Limaçon of Pascal [27], both trajectories of the second kind.

Acknowledgements We thank the referee for his/her careful review of the manuscript and his/her valuable comments and suggestions. Y.V. acknowledge support by the Dirección de Investigación y Desarrollo de la Universidad de La Serena, Grant No. PR18142. J.R.V. was partially supported by Centro de Astrofísica de Valparaíso.

Data Availability Statement This manuscript has no associated data or the data will not be deposited. [Authors' comment: This is a theoretical paper without associated data.]

Open Access This article is licensed under a Creative Commons Attribution 4.0 International License, which permits use, sharing, adaptation, distribution and reproduction in any medium or format, as long as you give appropriate credit to the original author(s) and the source, provide a link to the Creative Commons licence, and indicate if changes were made. The images or other third party material in this article are included in the article's Creative Commons licence, unless indicated otherwise in a credit line to the material. If material is not included in the article's Creative Commons licence and your intended use is not permitted by statutory regulation or exceeds the permitted use, you will need to obtain permission directly from the copyright holder. To view a copy of this licence, visit http://creativecomm ons.org/licenses/by/4.0/.

Funded by SCOAP ${ }^{3}$.

\section{References}

1. T. Kaluza, Zum Unitätsproblem der Physik, Sitzungsber. Königl. Preuss. Akad. Wiss., pp. 966-972 (1921)

2. O. Klein, Quantentheorie und fünfdimensionale relativitätstheorie, Zeitschrift für Physik, 1926

3. A. Font, S. Theisen, Introduction to string compactification. Lect. Notes Phys. 668, 101 (2005)

4. C.S. Chan, P.L. Paul, H.L. Verlinde, A Note on warped string compactification. Nucl. Phys. B 581, 156 (2000). arXiv:hep-th/0003236

5. F.R. Tangherlini, Schwarzschild field in $\mathrm{n}$ dimensions and the dimensionality of space problem. Nuovo Cim. 27, 636 (1963)

6. D.G. Boulware, S. Deser, Phys. Rev. Lett. 55, 2656 (1985). https:// doi.org/10.1103/PhysRevLett.55.2656

7. V.P. Frolov, D. Stojkovic, Particle and light motion in a space-time of a five-dimensional rotating black hole. Phys. Rev. D 68, 06401 (2003). arXiv:gr-qc/0301016

8. D.N. Page, D. Kubizňák, M. Vasudevan, P. Krtouš, Complete Integrability of Geodesic Motion in General Higher-Dimensional Rotating Black-Hole Spacetimes. Phys. Rev. Lett. (2007)

9. E. Hackmann, V. Kagramanova, J. Kunz and C. Lammerzahl, Analytic solutions of the geodesic equation in higher dimensional static spherically symmetric space-times, Phys. Rev. D 78 (2008) 124018 Addendum: [Phys. Rev. D 79 (2009) no.2, 029901]. arXiv:0812.2428 [gr-qc]
10. G.W. Gibbons, M. Vyska, The Application of Weierstrass elliptic functions to Schwarzschild Null Geodesics. Class. Quant. Grav. 29, 065016 (2012). arXiv:1110.6508 [gr-qc]

11. S. Guha, S. Chakraborty, P. Bhattacharya, Particle motion in the field of a five-dimensional charged black hole. Astrophys. Space Sci. 341, 445 (2012). [arXiv:1008.2650 [gr-qc]]

12. D. Kovacs, The geodesic equation in five-dimensional relativity theory of Kaluza-klein. Gen. Relat. Gravity 16, 645 (1984)

13. V. Kagramanova, S. Reimers, Analytic treatment of geodesics in five-dimensional Myers-Perry space-times. Phys. Rev. D 86, 084029 (2012). arXiv:1208.3686 [gr-qc]

14. S.S. Seahra, P.S. Wesson, Null geodesics in five-dimensional manifolds. Gen. Rel. Grav. 33, 1731 (2001). arXiv:gr-qc/0105041

15. S. Guha, P. Bhattacharya, Geodesic motions near a fivedimensional Reissner-Nordstroem anti-de Sitter black hole. J. Phys. Conf. Ser. 405, 012017 (2012)

16. P.A. Gonzalez, M. Olivares, Y. Vasquez, Bounded orbits for photons as a consequence of extra dimensions. Mod. Phys. Lett. A 32(32), 1750173 (2017). arXiv:1511.08048 [gr-qc]

17. S. Grunau, H. Neumann, S. Reimers, Geodesic motion in the fivedimensional Myers-Perry-AdS spacetime. Phys. Rev. D 97(4), 044011 (2018). arXiv:1711.02933 [gr-qc]

18. J. Chandler, M.H. Emam, Geodesic structure of five-dimensional nonasymptotically flat 2-branes. Phys. Rev. D 91(12), 125024 (2015). arXiv:1506.06054 [gr-qc]

19. R.S. Kuniyal, H. Nandan, U. Papnoi, R. Uniyal, K.D. Purohit, Strong lensing and observables around 5D Myers-Perry black hole spacetime. Mod. Phys. Lett. A 33(23), 1850126 (2018). arXiv:1705.09232 [gr-qc]

20. J.M. Maldacena, The Large N limit of superconformal field theories and supergravity. Int. J. Theor. Phys. 38, 1113 (1999). arXiv:hep-th/9711200

21. J.M. Maldacena, The Large $\mathrm{N}$ limit of superconformal field theories and supergravity. Adv. Theor. Math. Phys. 2, 231 (1998). arXiv:hep-th/9711200

22. E. Witten, Anti-de Sitter space and holography. Adv. Theor. Math. Phys. 2, 253 (1998). arXiv:hep-th/9802150

23. S.S. Gubser, I.R. Klebanov, A.M. Polyakov, Gauge theory correlators from noncritical string theory. Phys. Lett. B 428, 105 (1998). arXiv:hep-th/9802109

24. J.J. Friess, S.S. Gubser, G. Michalogiorgakis, S.S. Pufu, Expanding plasmas and quasinormal modes of anti-de Sitter black holes. JHEP 0704, 080 (2007). arXiv:hep-th/0611005

25. A. Chamblin, R. Emparan, C.V. Johnson, R.C. Myers, Charged AdS black holes and catastrophic holography. Phys. Rev. D 60, 064018 (1999). arXiv:hep-th/9902170

26. A. Chamblin, R. Emparan, C.V. Johnson, R.C. Myers, Holography, thermodynamics and fluctuations of charged AdS black holes. Phys. Rev. D 60, 104026 (1999). [hep-th/9904197]

27. J.R. Villanueva, J. Saavedra, M. Olivares, N. Cruz, Photons motion in charged Anti-de Sitter black holes. Astrophys. Space Sci. 344, 437 (2013)

28. H. Saadat, Thermodynamics and stability of five dimensional AdS Reissner-Nordstroem black hole. Int. J. Theor. Phys. 51, 316 (2012)

29. S. Chandrasekhar, The mathematical theory of black holes (Oxford University Press, Oxford, 2002)

30. N. Cruz, M. Olivares, J.R. Villanueva, The Geodesic structure of the Schwarzschild anti-de Sitter black hole. Class. Quant. Grav. 22, 1167 (2005). arXiv:gr-qc/0408016

31. J.R. Villanueva, F. Tapia, M. Molina, M. Olivares, Null paths on a toroidal topological black hole in conformal Weyl gravity. Eur. Phys. J. C 78(10), 853 (2018). arXiv:1808.04298 [gr-qc]

32. N. Cruz, M. Olivares, J.R. Villanueva, Geodesic Structure of Lifshitz Black Holes in 2+1 Dimensions. Eur. Phys. J. C 73, 2485 (2013). arXiv:1305.2133 [gr-qc] 
33. M. Olivares, G. Rojas, Y. Vásquez, J.R. Villanueva, Particles motion on topological Lifshitz black holes in $3+1$ dimensions. Astrophys. Space Sci. 347, 83 (2013). arXiv:1304.4297 [gr-qc]

34. J.R. Villanueva, Y. Vásquez, About the coordinate time for photons in Lifshitz Space-times. Eur. Phys. J. C 73(10), 2587 (2013). arXiv:1309.4417 [gr-qc]

35. P.A. González, M. Olivares, E. Papantonopoulos, Y. Vásquez, Motion and trajectories of photons in a three-dimensional rotating Hořava-AdS black hole. Phys. Rev. D 101(4), 044018 (2020). arXiv: 1912.00946 [gr-qc]

36. M. Robert, Wald (University Of Chicago Press, General Relativity, 1984)
37. C. Chirenti, A. Saa, J. Skakala, Quasinormal modes for the scattering on a naked Reissner-Nordstrom singularity. Phys. Rev. D 86, 124008 (2012). arXiv:1206.0037 [gr-qc]

38. D. Pugliese, H. Quevedo, R. Ruffini, Circular motion of neutral test particles in Reissner-Nordström spacetime. Phys. Rev. D 83, 024021 (2011). arXiv:1012.5411 [astro-ph.HE]

39. J. Dennis Lawrence, A catalog of special plane curves, Dover Publications, 1972

40. N. Straumann, General relativity and relativistic astrophysics (Springer, Berlin, 1984) 